\title{
Brain Network Analyzer
}

\author{
Vassilis Tsiaras $^{1,2}$, Ioannis G. Tollis ${ }^{1,2}$, and Vangelis Sakkalis ${ }^{1}$ \\ ${ }^{1}$ Institute of Computer Science, FORTH \\ ${ }^{2}$ Department of Computer Science, University of Crete \\ Heraklion, Greece \\ \{tsiaras, tollis, sakkalis\}@ics.forth.gr
}

\begin{abstract}
Brain Network Analyzer is an application, written in Java, that displays and analyzes synchronization networks from brain signals. The program implements a number of network indices and visualization techniques. The program has been used to analyze networks produced by electroencephalogram data of alcoholic and control patients.
\end{abstract}

\section{Introduction}

One of the major issues in neuroscience is to describe how different brain areas communicate with each other during perception, cognition, action as well as during spontaneous activity in the default or resting state. Data acquired using noninvasive techniques [like functional magnetic resonance imaging (fMRI); electroencephalography (EEG); magnetoencephalography (MEG)] may be used to estimate functional connectivity, which is defined as the statistical dependence between the activations of distinct and often well separated neuronal populations. Network models provide a common framework for describing functional connectivity. However defining what nodes and edges should be is a challenging problem. Network nodes can easily be identified with fMRI data but the dependence between nodes can be measured only for low frequencies $(<0.2 \mathrm{~Hz})$ due to the limited time resolution of fMRI. On the other hand the time resolution of EEG/MEG is excellent but the mapping from generators in the brain to the sensors on the scalp is complex and the topology of a network in sensors space is different from the topology in generators space [1]. To identify generators from EEG/MEG data one has to solve the inverse problem which is ill posed and sensitive to noise. In the following we assume that connectivity networks associated with EEG/MEG data have been defined as adjacency matrices.

\section{Program Presentation}

Brain Network Analyzer is a program that visualizes and analyzes functional connectivity networks as well as connectivity networks at sensors space. The input consists of a series of adjacency matrices $W_{n}, n=1,2, \ldots, T$. To improve the signal to noise ratio the user may use hard or soft thresholds. Using hard thresholds each matrix $W_{n}$ is transformed into a binary matrix with entries in $\{0,1\}$. 


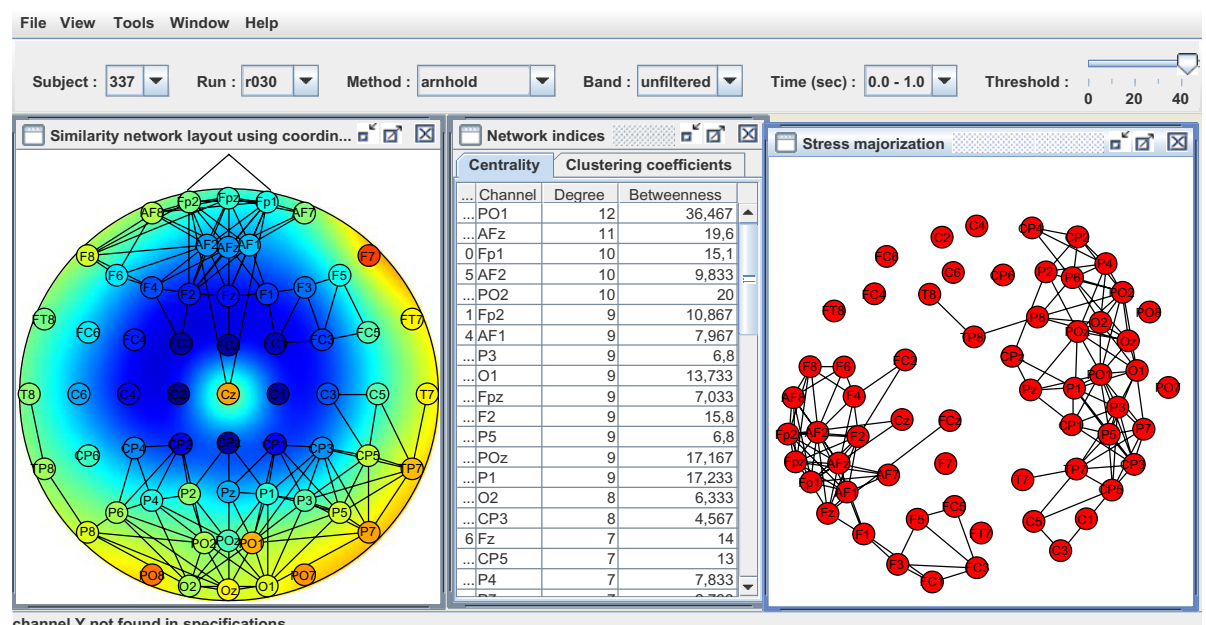

channel Y not found in specifications

Fig. 1. Synchronization network and topographic map are calculated at sensors space from EEG data. Network indices and multidimensional scaling windows are also shown.

Soft thresholds, such as the power function $f(w)=w^{\beta}$, are functions from $[0,1]$ to $[0,1]$. Entry $W_{n}(i, j)$ is a measure of how similar (or synchronous) is the dynamics of node $j$ to the dynamics of node $i$. To turn this similarity measure into a dissimilarity (or distance) measure we use the transformation $d(w)=-\log (w)$. Distance measures are needed for calculating multi-dimensional scaling and displaying, in the plane, nodes with similar functionality close together (see Fig. 11). In the program's menu there are options to visualize raw signals, potential maps, scalp topographies, and networks where node coordinates are defined either by the position of the generators in the brain or the sensors on the scalp or by an algorithm that groups functionally similar nodes. The user may also produce video with the evolution of networks during a cognitive task.

Finally, most of network indices, that appeared in complex networks literature, have been implemented. Using this program we found differences in clustering coefficient indices among the alcoholic and control subjects beta band (13-30 $\mathrm{Hz}$ ) networks at sensors space [2].

\section{References}

1. Ioannides, A.A.: Dynamic functional connectivity. Current Opinion in Neurobiology 17, 161-170 (2007)

2. Sakkalis, V., Tsiaras, V., Zervakis, M., Tollis, I.G.: Optimal brain network synchrony visualization: Application in an alcoholism paradigm. In: IEEE EMBS, pp. 42854288 (2007) 